\title{
Relevance of dynamic wetting in viscous fingering patterns
}

\author{
E. Álvarez-Lacalle, ${ }^{1}$ J. Ortín, ${ }^{2}$ and J. Casademunt ${ }^{2}$ \\ ${ }^{1}$ Department of Physics of Complex Systems, Weizmann Institute of Science, Rehovot 76100, Israel \\ ${ }^{2}$ Departament d'Estructura i Constituents de la Matèria, Universitat de Barcelona, Av. Diagonal 647, E-08028-Barcelona, Spain
}

(Received 26 July 2005; revised manuscript received 3 February 2006; published 29 August 2006)

\begin{abstract}
We demonstrate that wetting effects at moving contact lines have a strong impact in viscous fingering patterns. Experiments in a rotating Hele-Shaw (HS) cell, dry or prewetted, show consistent morphological differences. When the wetting fluid invades a dry region, contact angle dynamics yield a kinetic contribution to the interface pressure drop that scales with capillary number as $\mathrm{Ca}^{2 / 3}$ but is significantly larger than the Park-Homsy kinetic correction. Numerical results are in very good agreement with experiments and show that standard HS equations work best for prewetted cells.
\end{abstract}

DOI: 10.1103/PhysRevE.74.025302

\section{INTRODUCTION}

Contact line dynamics and wetting properties [1] in the presence of a morphological instability have been shown to have an important influence on pattern formation [2-6]. In this paper we focus on the effects of dynamic wetting in viscous fingering in a Hele-Shaw (HS) cell [7-9], a problem often regarded as a paradigm of interfacial pattern formation. In this case, the confinement of the fluids between the two close plates of the cell reduces the usual problem of a contact line plus a free surface, to a simpler one consisting of two contact lines tied together by a meniscus [10].

The influence of wetting on the steady-state selection problem in viscous fingering has been studied in detail (see, for instance, $[10,11])$. These studies have dealt with the influence of the coating layers that form when a nonwetting fluid displaces a wetting fluid. In the reverse situation when a wetting fluid displaces a nonwetting fluid, however, we show that the presence of moving contact lines plays a crucial role in the resulting pattern morphologies. This scenario is encountered typically in fingering instabilities driven by density contrast (by either gravity or centrifugal force).

\section{EXPERIMENTAL RESULTS}

We have performed two series of experiments in a rotating HS cell. The experimental setup and protocol are described in detail in Refs. [12-14]. The first series is performed in a dry cell, as in Ref. [12]. A circular drop of silicone oil is injected at the cell center, surrounded by air, and set to rotate without further injection, making the initial circular interface unstable. In the second series of experiments, the glass plates are initially coated with a thin layer of silicone oil, following the prewetting procedure described in Ref. [13].

We use silicone oils Rhodorsil 47V 500 and 47V 50, of dynamic viscosities $\mu=0.55$ and $0.052 \mathrm{~Pa}$ s, respectively, at room temperature, density $\rho=975 \pm 10 \mathrm{~kg} / \mathrm{m}^{3}$, and surface tension $\sigma=20.7 \mathrm{mN} / \mathrm{m}$. The experiments are performed with gap thickness $b=0.2,0.5$, and $0.8 \mathrm{~mm}$, angular velocities $\Omega=30,60,90,100,120,150 \mathrm{rev} / \mathrm{min}$, and initial radii $R_{0}$ from 4 to $10 \mathrm{~cm}$. Combinations of these parameters allow the generation of patterns in a wide range of time and length scales. Pattern morphologies are essentially controlled by the
PACS number(s): 47.20.Ma, 47.20.Hw, 47.54.-r, 45.70.Qj

dimensionless combination of experimental parameters $S$ $=R_{0}^{3} \Omega^{2} \rho / \sigma$, a ratio of centrifugal to capillary forces. $S$ determines a most significant feature of the patterns, the number of fingers in the early nonlinear regime $n \sim \sqrt{(1+S) / 3}$ [12]. The degree of development of the patterns is controlled by the dimensionless time $t / \tau$, where $\tau=12 \mu /\left(\rho \Omega^{2} b^{2}\right)$. The other relevant dimensionless parameter of the problem is the (local) capillary number $\mathrm{Ca}=\mu \vec{v} \cdot \hat{n} / \sigma$ (with $\hat{n}$ directed outwards), a ratio of viscous to capillary forces.

In Fig. 1, we display snapshots of two experiments with the same set of parameters, for dry and wet conditions. The dynamics are clearly sensitive to the wetting condition, and lead to remarkably different morphologies. We find that these differences are very systematic and reproducible in a wide range of experimental parameters ( $n$ from 5 to $30, t / \tau$ from 0.5 to 1.5 , typically). The main features are well captured by the representative examples of Figs. 2 and 3. The typical morphology in the wet case produces fingers elongated and thin, with droplet-shaped ends. The longer fingers escape from the rest, with a nearly exponential growth of the radial distance [15]. For the dry case, instead, the most prominent fingers are slowed down, and pile up into a front with a nearly circular envelope, with a slowly growing radius. This is to be compared with the more polygon-shaped convex envelope of the wet case. In addition, the few air fingers that manage to penetrate deeply into the liquid tend to adopt a typical balloonlike shape, close to circular.

A quantitative analysis of the temporal evolution of experiments is presented in Fig. 4. We have singled out for each experiment one of the most prominent outgoing fingers, and measured the dimensionless radial coordinate of the tip, $r_{+}(t) / R_{0}$. This clearly discriminates between wet and dry cases. We find an excellent collapse of all the experiments performed in wet conditions. The experiments in dry conditions, although not showing such a good collapse, do exhibit the systematic slowing down of the pattern growth.

It is important to note that, in a dry cell, different parts of the same interface meet regions of the cell that may be either wet or dry. The wet regions are those previously visited by the oil, so, strictly speaking, the local wetting conditions will depend on the whole history of the flow. In practice, it is sufficient to assume that the points of the interface with positive (negative) normal velocity meet dry (wet) regions of the cell [16]. 

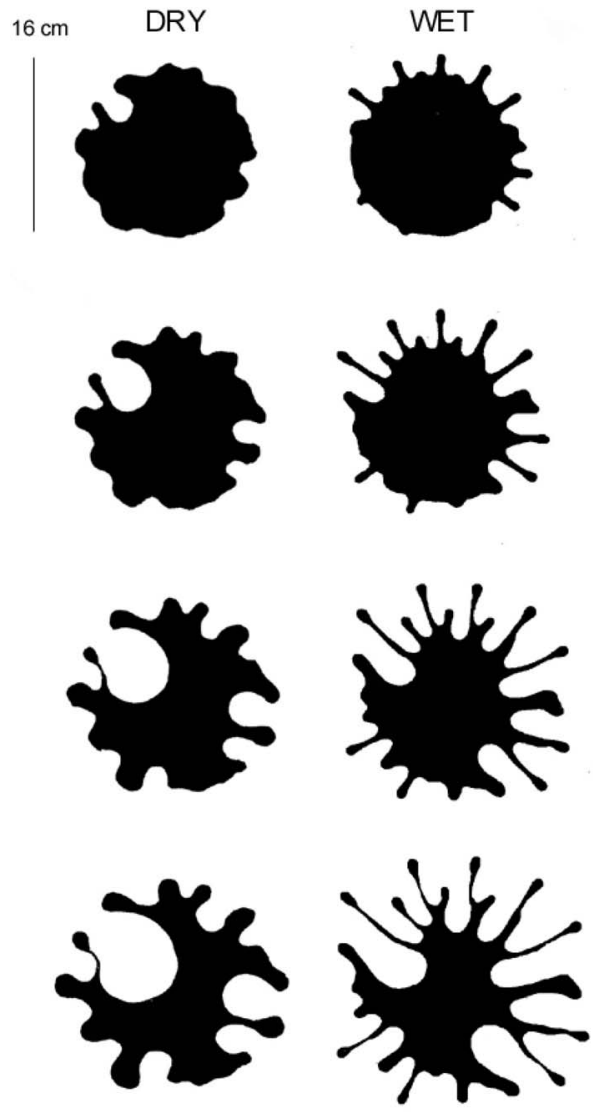

FIG. 1. Time sequence of two experiments performed in a dry cell (left) and in a prewetted cell (right) for the same set of parameters. The time lapse between consecutive snapshots is $9 \mathrm{~s}$. The parameters are $b=0.5 \mathrm{~mm}$ and $\Omega=60 \mathrm{rev} / \mathrm{min}$. The initial radii, $R_{0}=7.6 \mathrm{~cm}$ (left) and $7.9 \mathrm{~cm}$ (right), are the same within experimental error because the initial drop is not perfectly circular, so that $S \simeq 7 \times 10^{4}$. Maximal values of $\mathrm{Ca}$ (for the fastest fingertips in the last snapshot) are $3.3 \times 10^{-3}$ (left) and $4.2 \times 10^{-3}$ (right).

\section{THEORETICAL DISCUSSION}

The effective two-dimensional (2D) velocity of an incompressible fluid in the cell, $\vec{v}$, satisfies $\vec{v}=\vec{\nabla} \phi$, with $\vec{\nabla} \cdot \vec{v}=0$ and $\phi=-\frac{b^{2}}{12 \mu}(p-F)$, where $p$ is the pressure and, for a rotating HS cell, $F=\frac{1}{2} \rho \Omega^{2} r^{2}$ with $r$ the radial coordinate. The boundary conditions commonly used in the literature are the continuity of the normal velocity, and the equilibrium Laplace-Young pressure drop for the 2D meniscus $p_{2}-p_{1}$ $=\sigma(\kappa-2 \cos \theta / b)$, where the label 1 (2) refers to the outer (inner), less (more) viscous fluid, $\kappa$ is the curvature in the cell plane (taken positive for a circle), and $2 \cos \theta / b$ is the curvature perpendicular to the plane, where $\theta$ is the contact angle.

For a nonwetting fluid (air) displacing a wetting one (oil) (as for the receding parts in our experiment and for the prewetting procedure), Park and Homsy [17] have shown that the Laplace-Young pressure-drop boundary condition is actually the lowest-order approximation in a double expansion on $\mathrm{Ca}$, and $\epsilon=b / L$, where $L$ is the typical scale of transverse variation. Then, the effect of the dynamic formation of the coating layers gives, for the projected 1D interface,

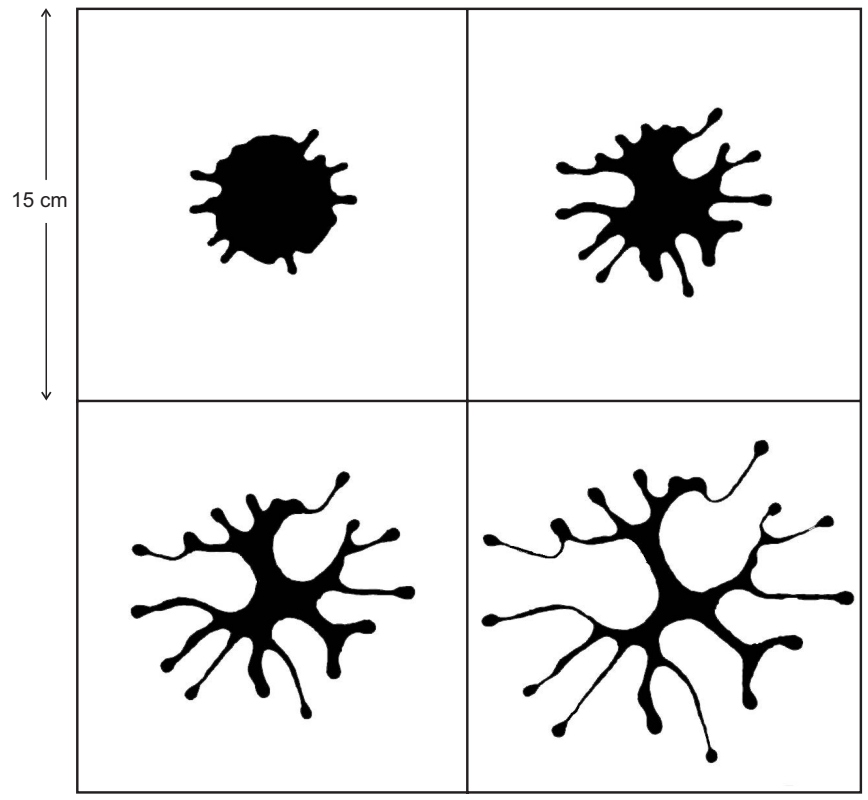

FIG. 2. Time sequence of fingering patterns formed in a wet cell

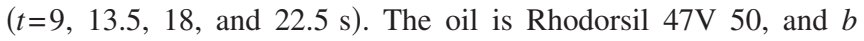
$=0.5 \mathrm{~mm}, \Omega=120 \mathrm{rev} / \mathrm{min}, R_{0}=5 \mathrm{~cm}\left(S=2.3 \times 10^{5}\right)$. The maximal $\mathrm{Ca}$ is $1.6 \times 10^{-2}$.

$$
p_{2}-p_{1}=\sigma\left[K-\frac{2}{b}\left(1+J|\mathrm{Ca}|^{2 / 3}\right)\right]
$$

with $K=\frac{\pi}{4} \kappa+O\left(|\mathrm{Ca}|^{2 / 3}\right)$ and $J=3.80+O\left(|\mathrm{Ca}|^{1 / 3}\right)$. The curvature term is assumed to be small (of order $\epsilon^{2}$ ) so the kinetic corrections to $K$ are then of order $\epsilon^{2}|\mathrm{Ca}|^{2 / 3}$.

In the prewetting procedure, the oil filling the cell is displaced by air at $\mathrm{Ca}_{0}[18]$, forming coating layers of thickness

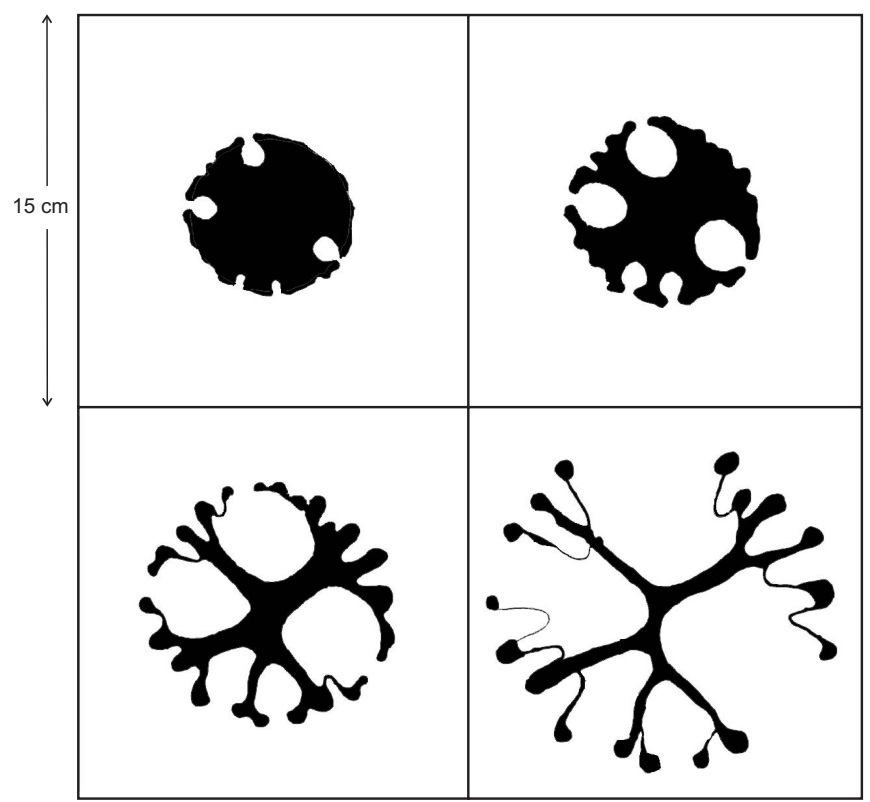

FIG. 3. Time sequence of fingering patterns formed in a dry cell $(t=8.4,10.4,14.4$, and $18.4 \mathrm{~s})$. The oil is Rhodorsil 47V 50, and $b=0.5 \mathrm{~mm}, \Omega=150 \mathrm{rev} / \mathrm{min}, R_{0}=6 \mathrm{~cm}\left(S=6.6 \times 10^{5}\right)$. The maximal $\mathrm{Ca}$ is $1.7 \times 10^{-2}$. 


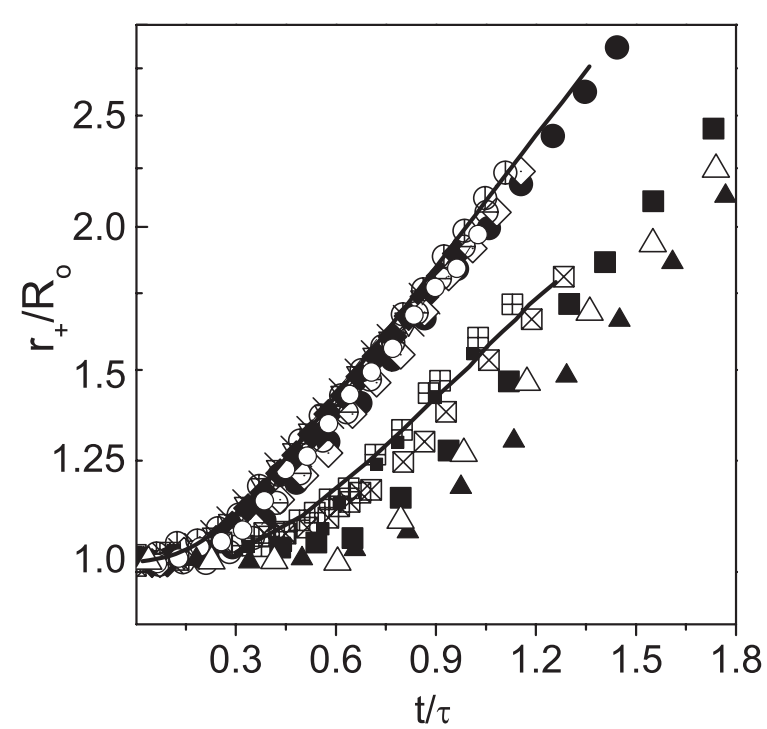

FIG. 4. Dimensionless maximum radial coordinate of outgoing fingers $r_{+} / R_{0}$ vs dimensionless time $t / \tau$ in log-linear scale, for wet (circles and diamonds) and dry (squares and triangles) conditions. The two solid lines are the numerical results for the corresponding cases. See text for details. The range of maximal $\mathrm{Ca}$ is $(1.0$ $\left.\times 10^{-3}\right)-\left(2.5 \times 10^{-2}\right)$.

$d \simeq 0.67 b\left|\mathrm{Ca}_{0}\right|^{2 / 3}[19]$ (always below $3 \%$ of $b$ in our experiments). Then, in the subsequent experiment, for the regions where oil is invading the coated plates, and extending the analysis of Bretherton [19], Eq. (1) applies with $J=J(\eta)$, an unknown function of $\eta \equiv \mathrm{Ca} / \mathrm{Ca}_{0}$, which must be determined numerically from asymptotic matching. Reference [19] shows that $J(\eta)>0$ for small $\eta$ and decreases monotonically, crossing zero around $\eta \simeq 0.4$. In the range $1 \leqq \eta \lesssim 10$ we find $-1 \gtrsim J(\eta) \gtrsim-3$. However, as shown below, the term proportional to $J$ in Eq. (1) is negligible as long as $\mathrm{Ca}_{\max }$ and $\mathrm{Ca}_{0}$ are within the same order of magnitude, as in our experiments.

On the contrary, for a dry cell, the presence of a moving contact line (i.e., when the two fluids make contact with the glass plates) does have an important effect. We model this case by taking the contact angle as a dynamic variable. Ngan and Dussan [20] have pointed out that the variability of apparent dynamic contact angle with $\mathrm{Ca}$ can indeed be rather large in a HS cell even for liquids with good wetting properties. One reasonable approximation for small contact angles is $[6,21,22]$

$$
\theta^{3}=l \mathrm{Ca}, \quad l=9 \ln \frac{Y}{Y_{\infty}},
$$

where we have already assumed that, as for silicone oil, the static contact angle is zero. $Y$ is a macroscopic scale associated with the gap thickness $b$, and $Y_{\infty}$ is a microscopic cutoff length scale below which macroscopic hydrodynamics fails (for the silicone oil Rhodorsil 47V 50, $Y_{\infty} \simeq 60 \mathrm{~nm}$ [6]). Expanding $\cos \theta$ in the Laplace-Young expression for the pressure drop, and picking up the same $\pi / 4$ prefactor for $\kappa$ as in [17], we find, to lowest order,

$$
p_{2}-p_{1}=\sigma\left[\frac{\pi}{4} \kappa-\frac{2}{b}\left(1-\frac{1}{2}(l \mathrm{Ca})^{2 / 3}\right)+\cdots\right]
$$

at those points where the wetting fluid is invading a dry region of the cell $(\mathrm{Ca}>0)$. Thus the leading kinetic correction from dynamic wetting also scales as $\mathrm{Ca}^{2 / 3}$ but is significantly larger than for the typical advancing regions in the prewetted case. Taking $Y \simeq b$, we get $l \simeq 80-100$, i.e., $\frac{1}{2} l^{2 / 3}$ $\simeq 10$, to be compared with $|J(\eta)|$, which at most reaches 3 at the fastest points and at the late stages.

Both for theoretical insight and numerical simulation, it is convenient to consider the vortex-sheet formulation of the HS problem [8]. The evolution of the interface, $(d \vec{r} / d t) \cdot \hat{n}$ $=\vec{U} \cdot \hat{n}$, reads

$$
\vec{U}(s, t)=\frac{1}{\pi} \mathrm{P} \int \frac{\hat{z} \times\left[\vec{r}(s, t)-\vec{r}\left(s^{\prime}, t\right)\right]}{\left|\vec{r}(s, t)-\vec{r}\left(s^{\prime}, t\right)\right|^{2}} \gamma\left(s^{\prime}, t\right) d s^{\prime},
$$

which is defined uniquely by the interface position and the vorticity $\gamma$ at the interface,

$$
\gamma=B \Upsilon_{s}+\overrightarrow{\mathbf{U}} \cdot \hat{\mathbf{s}}-C \overrightarrow{\mathbf{r}} \cdot \hat{\mathbf{s}},
$$

where $s$ is the arclength, $B=\sigma b^{2} /(12 \mu), C=\rho \Omega^{2} b^{2} /(12 \mu), \hat{s}$ is the tangent vector, and the subscript $s$ denotes a derivative. Neglecting kinetic corrections, $Y=\frac{\pi}{4} \kappa$. We will refer to this case as the standard boundary condition. Within this formulation, our model for contact line motion reduces to

$$
\mathrm{Y}=\frac{\pi}{4} \kappa-\frac{2}{b} \cos \left[(l \mathrm{Ca})^{1 / 3}\right]
$$

\section{NUMERICAL INTEGRATION}

We have numerically solved the HS equations for a rotating cell, using the code described in Ref. [23]. The initial condition is a circle, modulated by the first 30 harmonic modes with an amplitude of $0.002 R_{0}$ and random phases.

In a first series of computations, we use the standard boundary condition, $Y=\frac{\pi}{4} \kappa$. Remarkably, our numerical results systematically show good agreement with experiments in the wet cell. First, Fig. 4 shows good quantitative agreement for the evolution of $r_{+} / R_{0}$. Second, the morphologies found in this case mimic well the experimental ones. Snapshots of the late stages of the evolution in two representative cases are shown in Fig. 5. These results show that neither the kinetic correction $J|\mathrm{Ca}|^{2 / 3}$ nor the (unknown) corrections of $O\left(\epsilon^{2}|\mathrm{Ca}|^{2 / 3}\right)$ in Eq. (1) (which could be relevant since $\epsilon \sim 1$ in the tip regions) is significant in our experiments. A proper numerical integration of all kinetic corrections in this case is indeed much more involved and is not addressed here [24].

A second series of computations has been designed to reproduce the experimental results in a dry cell. We have used the standard boundary condition for the receding regions $(\mathrm{Ca}<0)$ and Eq. (6) for the invasion of dry regions $(\mathrm{Ca}>0)$ [25]. Numerical results are shown in Figs. 4 and 5. The typical morphologies obtained are strongly resemblant to those observed in experiments in dry cells. Figure 5 is particularly illustrative because it allows a local comparison for identical initial conditions. 

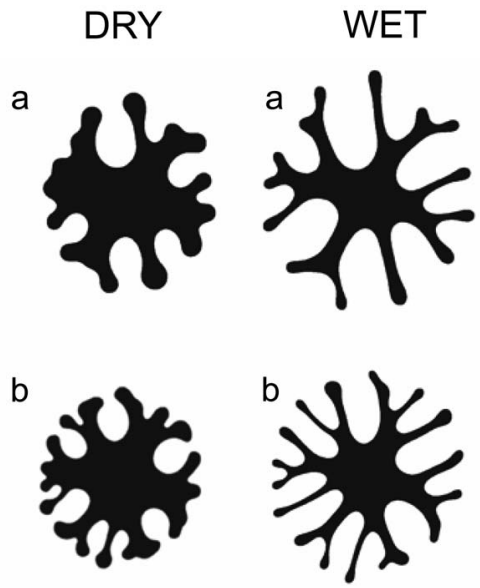

FIG. 5. Patterns from numerical integration, using the liquid

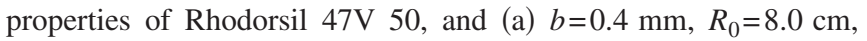
$\Omega=40 \mathrm{rev} / \mathrm{min} \quad\left(S=2 \times 10^{4}\right), \quad t=210 \mathrm{~s} ; \quad$ (b) $b=0.4 \mathrm{~mm}, \quad R_{0}$ $=10.0 \mathrm{~cm}, \Omega=60 \mathrm{rev} / \mathrm{min}\left(S=1.2 \times 10^{5}\right), t=140 \mathrm{~s}$. $Y$ given by Eq. (6) with $l=100$ has been used on the left panels, and the standard boundary condition on the right ones. The maximal $\mathrm{Ca}$ are 1.1 $\times 10^{-3}$ [wet (a)], $8 \times 10^{-4}$ [dry (a)], $2.0 \times 10^{-3}$ [wet (b)], 1.2 $\times 10^{-3}[$ dry (b) $]$.

\section{DISCUSSION AND CONCLUSIONS}

Experiments in a wet cell are typically well reproduced with the standard HS boundary conditions, implying that kinetic corrections have negligible impact on the morphologies in the range of capillary numbers explored $\left(\mathrm{Ca} \leq 10^{-2}, \mathrm{Ca}\right.$ $\left.\lesssim 10 \mathrm{Ca}_{0}\right)$.

Experiments in a dry cell show the important role of the dynamic contact angle. This can be properly taken into account through a modified boundary condition. In this case, the receding parts of the interface see the same conditions as in a wet cell, while the motion of the advancing parts is significantly slowed down by the presence of the contact lines. This different behavior has a strong impact on the resulting morphologies. In particular, the nearly circular envelope of the patterns can be understood on the basis of Eq. (6) in the following terms. The tendency of surface tension to decrease the curvature variation $\kappa_{s}$ of the interface is replaced by a tendency to decrease $Y_{s}$, i.e., to have an interface of uniform curvature and normal velocity. While the kinetic contribution to $Y$ could eventually stabilize the circular shape in the more traditional case of a radial HS cell with fluid injection, in the purely centrifugal case a circular shape is necessarily stationary $(\mathrm{Ca}=0)$ so that the kinetic contribution is not relevant at the early stages. However, when the fingering morphology is well developed, the circular shape can be roughly recovered in the envelope of the pattern. This suggests that the fingered region can be treated to some extent as an effective medium with lower (average) density. In such a case, the velocity of the circular envelope is not constrained by conservation, and the stabilization due to dynamic wetting can indeed be effective.

As a final comment, we would like to stress the remarkable fact that, in the case of the dry cell, a microscopic length scale (nanometer range) enters the macroscopic description of the problem, producing significant effects at the macroscopic scale (millimeter range), about six orders of magnitude apart.

\section{ACKNOWLEDGMENTS}

We are grateful to J. Eggers for fruitful discussions and to E. Pauné for numerical advice. This work has been partially supported by the European Commission, project HPRN-CT2002-00312, the MCyT (Spain), project BQU2003-05042C02-02, and the DURSI (Catalonia), project 2005-SGR00507.
[1] P. G. de Gennes, Rev. Mod. Phys. 57, 827 (1985).

[2] F. Melo et al., Phys. Rev. Lett. 63, 1958 (1989).

[3] S. M. Troian et al., Phys. Rev. Lett. 62, 1496 (1989).

[4] S. Kalliadasis, J. Fluid Mech. 413, 355 (2000).

[5] J. A. Diez and L. Kondic, Phys. Rev. Lett. 86, 632 (2001).

[6] T. Podgorski et al., Phys. Rev. Lett. 87, 036102 (2001).

[7] D. Bensimon et al., Rev. Mod. Phys. 58, 977 (1986).

[8] G. Tryggvason and H. Aref, J. Fluid Mech. 136, 1 (1983).

[9] J. Casademunt, Chaos 14, 809 (2004).

[10] S. J. Weinstein et al., J. Fluid Mech. 221, 53 (1990).

[11] P. Tabeling et al., J. Fluid Mech. 177, 67 (1987).

[12] Ll. Carrillo et al., Phys. Rev. E 54, 6260 (1996).

[13] Ll. Carrillo, J. Soriano, and J. Ortín, Phys. Fluids 11, 778 (1999); 12, 1685 (2000).

[14] E. Alvarez-Lacalle et al., Phys. Fluids 16, 908 (2004).

[15] The exponential growth of the radial coordinate is an exact result for an isolated droplet in a rotating cell, for the standard HS equations. See R. Folch et al., e-print physics/0408092.

[16] In principle, points of positive normal velocity may encounter regions previously visited by the oil. In practice, however, this is extremely rare.
[17] C. W. Park and G. M. Homsy, J. Fluid Mech. 139, 291 (1984).

[18] The velocity of the circular front in the prewetting procedure is essentially constant [13], giving rise to coating layers of nearly uniform thickness [17,19].

[19] F. P. Bretherton, J. Fluid Mech. 10, 166 (1961).

[20] C. G. Ngan and E. B. Dussan V, J. Fluid Mech. 118, 27 (1982); 209, 191 (1989).

[21] R. G. Cox, J. Fluid Mech. 168, 169 (1986).

[22] E. B. Dussan V et al., J. Fluid Mech. 230, 97 (1991).

[23] E. Pauné, Ph.D. thesis, University of Barcelona (2002).

[24] Overestimating the kinetic correction, by taking a constant $|J|$ equal to its overall maximum, produces patterns that are still much closer to those obtained with standard boundary conditions than those for the dry case.

[25] Around $\mathrm{Ca} \simeq 0$, the term $\left(\cos \left[(l \mathrm{Ca})^{1 / 3}\right]\right)_{s}$ is written as $-(1 / 3) l^{1 / 3} \mathrm{Ca}^{-2 / 3} \sin \left[(l \mathrm{Ca})^{1 / 3}\right] \mathrm{Ca}_{s} . \mathrm{Ca}_{s}$ is computed implicitly and the rest explicitly. Different ways to handle the integrable singularity have been tested without significant changes in the results. 